\title{
LA LITERATURA INFANTIL Y LAS NARRATIVAS HÍBRIDAS. CANON, MODOS DE PENSAMIENTO Y EDUCACIÓN
}

\author{
Eduardo Encabo Fernández \\ Lourdes Hernández Delgado \\ Gabriel Sánchez Sánchez \\ Universidad de Murcia
}

RESUMEN: El objetivo de esta aportación es describir y reflexionar sobre la Literatura infantil, la lectura y el contexto actual en el que tienen lugar. La presencia de formatos audiovisuales implica la coexistencia de estos con los textos escritos motivando un determinado comportamiento en el acceso al conocimiento. El análisis de una competencia enciclopédica posiblemente fragmentada frente a la competencia literaria será objeto de discusión. Dicha competencia incidirá en un probable nuevo modo de pensamiento y, sobre todo, en la pregunta referida a, si con estas circunstancias, existe la necesidad de un canon literario. Se concluye la necesidad de un planteamiento educativo que integre tanto los formatos físicos como los audiovisuales, así como la posibilidad de contemplar otro tipo de textos como fuente de conocimiento para las nuevas generaciones. Su formación inicial basada en percepciones audiovisuales y, sobre todo, en los hipertextos motiva que el profesorado deba plantearse este tipo de cuestiones en lo que concierne a los textos a seleccionar y utilizar y en lo que respecta al modo de enseñar.

PALABRAS CLAVE: Literatura infantil, alfabetización, educación, cultura.

\section{CHILDREN'S LITERATURE AND HYBRID NARRATIVES. CANON, WAYS OF THINKING AND EDUCATION}

\begin{abstract}
The main aim of this contribution deals with how to describe and reflect on Children's literature, reading and the context in which they are taught. One of the most important ideas is the coexistence of audiovisual formats with written texts which implies a concrete way within the learning process and the knowledge acquisition. The analysis of a partial encyclopaedic competence versus literary competence will be discussed in the text. This competence will bring a new way of thinking and, above all, it will bring about
\end{abstract}


an important question regarding whether the literary canon is necessary or not. The major conclusion is that a new educative approach is needed, one which either includes written and audiovisual formats as a source of knowledge for new generations. Their initial formation on literacy is based on the audiovisual and, above all, on their use of hypertexts; something which means that teachers must bear in mind this kind of issues in order to select and use literary texts in the classroom.

KEYWORDS: Children's literature, literacy, education, culture.

Recibido: 10/12/2017

Aceptado: 09/02/2018

Correspondencia: Eduardo Encabo Fernández, Universidad de Murcia, C/ Campus Universitario, s/n, 30100 Murcia. Email: edencabo@um.es.

\section{INTRODUCCIÓN}

En este texto planteamos los cambios sociales que afectan a la Literatura infantil, transformaciones que probablemente alberguen una percepción distinta de lo que realmente acontece. Parece ser que en la era tecnológica, los elementos relacionados con la lectura pudiesen verse afectados, motivando un cambio de paradigma en lo que concierne al acceso al conocimiento o información.

La globalización y ante todo, la homogeneización económica han conllevado que se promulguen patrones de comportamiento que implican la proliferación de materiales audiovisuales que compiten con el texto impreso tradicional. También hay que precisar que, aunque acontezca dicha irrupción, también es cierto que las editoriales siguen publicando gran cantidad de libros y materiales relacionados con la Literatura infantil. Un dato importante al respecto nos lo proporciona la Federación de Gremios de Editores de España (2018) cuando nos indica que el 61.3\% de la población española de 14 o más años ha comprado algún libro ya sea de texto o no de texto en los últimos 12 meses. Existe una notable diferencia entre la promoción lectora que se preconizaba hace una década (García Padrino, 2005) y las posibilidades actuales que la sociedad presenta a los mediadores de la lectura. La misma Federación anteriormente reseñada nos proporciona el dato concerniente a que el $76.3 \%$ de los españoles a partir de los 14 años (58.0\% en 2012) lee algún tipo de contenido en soporte digital. Por ello, nos atrevemos a reflexionar sobre la combinación que relaciona la noción de crisis lectora con el auge audiovisual en el ámbito de la disciplina estudiada.

La coexistencia de ambas manifestaciones, la impresa y la digital, nos conduce a cavilar sobre el tipo de pensamiento que desarrollan tanto las generaciones que se encuentran formándose con ambos formatos, así como los educadores que se hallan implicados en ese proceso. Parece evidente que la forma de tratamiento de la información cuando a esta se accede por distintas vías, varía, y lo hace propiciando que el modo de pensar quede modificado. 


\section{LA POSIBLE CRISIS LeCtORA Y LA INTUIDA PREVALENCIA DEL SECTOR AUDIOVISUAL}

La pasada década y la actual han mostrado una preocupación social por los índices de participación lectora que muestran los jóvenes y las personas adultas, ya que, si lo circunscribimos al ámbito educativo, van a ser ellas las que influyan y sirvan como modelo para los más pequeños. Son frecuentes las noticias en los medios de comunicación de masas en los que se alerta sobre el porcentaje de lectores habituales, pese a que la Federación de Gremios de Editores de España (2018) nos dice que el $65.8 \%$ de la población española lee libros, un 59.7\% por ocio, en su tiempo libre, y el $28.1 \%$ por motivos de trabajo o estudio. Probablemente tengamos que cuestionar esas cifras por mor de la posible corrección política presente en las respuestas y considerar ese presunto descenso de usuarios de la lectura; también hallamos publicaciones como las de Cerrillo (2016) o Basanta (2017) que tratan de profundizar en el concepto de los lectores actuales y sobre cómo afrontar el hecho lector. Tales divulgaciones no dejan de responder sino a una foto fija que, dependiendo del medio que difunda la información, será más o menos sensacionalista. En cualquier caso, nos encontramos en un impás social en el que la idea de crisis lectora se halla instaurada en el imaginario colectivo. Esta noción afecta al comportamiento de las personas, ya que, van a mostrar una actitud políticamente correcta hacia la lectura -pocas personas expresaran su disconformidad o rechazo- pero tampoco se apreciará un gusto por la lectura y un aprovechamiento de esta. Desde nuestro punto de vista, no conviene ser categóricos a la hora de optar por posicionamientos que atañan a la lectura. Así, aquellas opiniones que vinculan los pilares de la cultura a la prevalencia del texto escrito y a la necesidad de adquirir y cultivar el conocimiento, olvidan o dejan de lado la influencia que poseen otros formatos o fuentes que, sin duda también proporcionan información y van configurando el pensamiento.

Tampoco la actitud de desdén hacia la lectura colabora demasiado en la conformación del pensamiento y, ante todo, en la completa inclusión social de las personas. Como aspecto tradicional de acceso a la información, la lectura se encuentra instaurada socialmente como una herramienta o una convención que desde los sistemas educativos se proporciona a las diferentes generaciones. Aportaciones como las Alonso (2015) reflexionan en torno a la idea de la lectura y la literatura en el contexto escolar y la trascendencia social que esta puede poseer. No profundizar en el hecho lector, en su uso, implica desaprovechar posibilidades del pensamiento humano y, principalmente, no poder acceder a información que permite conocer más sobre la realidad y sobre el mundo -tanto en una dimensión sincrónica como en la diacrónica-. Las progresivas transformaciones sociales hacen que pueda existir la percepción de crisis lectora pero lo categórico tomado como referencia puede inducir a error en el comportamiento humano.

Uno de los factores que se vislumbra como desencadenante de la crisis lectora que se indica se detecta en la sociedad, es el auge audiovisual. Ya hemos señalado en la introducción el porcentaje de usuarios de contenido en soporte digital que existe en España. La irrupción de los medios de comunicación de masas, comandados sobre todo, por la televisión e internet, ha modificado de forma significativa los com- 
portamientos de las personas. El aspecto que concierne a la comodidad, encontrando el ser humano información rápidamente y sin demasiado esfuerzo a través de la red o de la producción de programas televisivos. Esta situación motiva que podamos tildar la situación social actual como hecho cultural donde prevalece el sector audiovisual, es decir, la información fluye mediante estos medios, y el hecho lector queda eclipsado. Aparentemente, esto puede ser así. Es más, si en la formación de la persona, la lectura queda relegada a ser un instrumento, su valor referido al conocimiento decaerá y se banalizará. Esta última situación conllevará la pérdida de importancia del acto lector y equiparará la función de acceso a la información de los distintos formatos.

Una vez realizada la similitud, es lógico pensar que las personas optarán por la comodidad del formato audiovisual en virtud del ahorro de tiempo y esfuerzo. En nuestra opinión la prevalencia audiovisual debe quedar como una intuición, ya que, no es real que todo quede supeditado a estos formatos. La lectura sigue teniendo su espacio, únicamente hay que tratar $-\mathrm{y}$ en el caso del mundo educativo es primordialde hacer visible su importancia y, sobre todo, poder generar la misma satisfacción que el medio audiovisual consigue en la persona. Sentir que la lectura de un texto ha recompensado al individuo supone un estímulo fundamental para poner en valor el hecho y para equilibrar cuestiones tradicionales con elementos más modernos que conviven diariamente con las personas. Además, debemos tener en cuenta a los translectores, cada vez más, que hacen uso de la lectura tanto en papel como en la web (Jenkins, 2008; Edwards, 2012; Koenitz y otros, 2015; Scolari, 2017). En el siguiente apartado, reflexionaremos sobre la posible coexistencia de ambos formatos, procurando de ese modo un mayor beneficio para la formación de la persona y para su bienestar personal y como miembro de un entramado social.

\section{LA PRETENDIDA COEXISTENCIA ENTRE El TEXTO ESCRITO Y LOS FORMATOS AUDIOVISUALES}

Desde estas líneas optamos por un enfoque equilibrado entre la tradición y la contemporaneidad. En el primero de los casos, como hemos expresado previamente, consideramos que el concepto lector como aspecto que hace evolucionar al ser humano tanto en su individualidad como en su colectividad, debe ser mantenido. La lectura permite conectar distintos contenidos que se relacionan directamente con la experiencia, y lo que es más importante, la presencia del factor ficción motiva que la persona pueda crear una visión única y particular a la vez que vivenciar aspectos que son propiamente humanos pero que no tienen por qué estar atribuidos a ella en primera persona sino que mediante un personaje no real, es posible identificar valores, pensamientos o deseos que sí forman parte de la existencia humana pero, que, a través de la ficción, se camuflan, sin perder por ello su valor formativo o su poder de satisfacción.

La aproximación a una lectura o a un texto requiere de una motivación. Esta no es sencilla de hallar, ya que, los efectos placenteros o formativos del acto lector no son inmediatos. De ahí que las estrategias de motivación o de convencimiento sean esenciales para poder mantener el interés por esta habilidad humana y mantenerla como un bastión cultural. En aportaciones como las de Quiles, Palmer y Rosal (2015) o la 
de Martínez Ezquerro (2016) podemos encontrar estrategias de acceso a la educación literaria que recogen la esencia de ese espíritu motivador y creador de hábito que es tan necesario para la formación lectora de la persona. Como se ha mencionado en el apartado precedente, al lectura no corre peligro social en tanto que puede mantenerse de manera utilitaria, pero si queremos que se mantenga como un eje generador y constructor de conocimiento, sí que tendremos que procurar las estrategias de comprensión y promoción que permitan su reconocimiento como elemento esencial vital.

En lo que respecta al otro componente de la balanza, es importante remarcar que la cotidianidad con la que se accede a los medios tecnológicos es ineludible y por esa razón los formatos audiovisuales son portadores de información social y cultural que redundan en la conformación del pensamiento de la persona. ¿Son incompatibles con la acción lectora? Desde nuestra óptica no. Ya que lo que se modifica es el formato en el que se transmite la información. Por ello, el reto estriba en hacer ver a las personas que no es obligatoria la elección entre uno de los dos formatos presentados sino que es posible la alternancia y, sobre todo, la concomitancia. Una persona que es capaz de filtrar información audiovisual y ponerla en contacto con su experiencia lectora, lógicamente posee un bagaje experiencial más rico y, por ende, tendrá un conocimiento más comprensivo de su realidad.

En el caso de la Literatura infantil, la necesidad del equilibrio es más significativa porque, cercanos a la tercera década del siglo veintiuno, las generaciones que van a ser usuarias de dicha disciplina, van a ser personas que podrán gozar de la condición de ser nativos digitales. Su manejo de los medios audiovisuales será muy bueno, teniendo los educadores la responsabilidad de introducirlos en el mundo lector mediante la utilización de los textos en formato físico y otros formatos, y sobre todo, a través del uso de lecturas reales que no sean oscurecidas por adaptaciones, hibridaciones o simplificaciones de historias que estos niños puedan conocer. El reto es importante, ya que la lectura tradicional posee una desventaja clara con respecto de los formatos audiovisuales, esto es, no tiene inmediatez y no es tan Ilamativa como la imagen o los efectos sonoros y de animación pero dan visiones únicas y personales de los personajes y acontecimientos narrados. Ejemplos de estas reflexiones los hallamos en aportaciones como la de Sánchez Morillas (2013) donde se trata la situación de los personajes clásicos en un papel distinto del de de su creación, en un contexto temporal actualizado donde las acciones se corresponden con una época que es en la que los niños viven. Ahora bien, de cómo un mediador aproxime una lectura a los niños va a depender el interés y la comprensión relativa a la importancia de leer de una manera comprensiva en las edades más tempranas. Como veremos a continuación, en la formación de los niños, podemos optar por permitir que se provean de información procedente de medios parcelados con lo que de ese modo tendrán una formación cultural algo segada o podemos intentar hacer que su competencia literaria se desarrolle y sea complementada con una competencia enciclopédica que contextualice determinados aspectos en los que se apoya la literatura para dar sentido a su desarrollo ficcional. 


\section{LA COMPETENCIA ENCICLOPÉDICA POSIBLEMENTE FRAGMENTADA FRENTE A LA COMPETENCIA LITERARIA}

Como hemos reseñado, las condiciones sociales propician que la información que contienen los formatos audiovisuales fluya de una manera más rápida que el acceso que se pueda tener a través del manejo de un libro. Pero dicha información procedente de lo audiovisual corre el riesgo de dispersarse, ya que, en la mayoría de las ocasiones es ofrecida de un modo sesgado o fragmentado. Esa situación unida a las modificaciones de los textos o de la información que a veces se produce, motiva que el conocimiento adquirido por las personas se constituya en meramente enciclopédico, pero tampoco esta situación implica que esa cultura recibida o conocida esté completa o se ajuste a la información. Así, por ejemplo, es frecuente apreciar en algunos educadores y docentes que trabajan con niños la falta de referencias sobre el origen y autores de los cuentos clásicos que suelen usar. La factoría Disney/Pixar mediante sus adaptaciones, ha logrado que, para estos educadores, el referente sea una marca y que atribuyan a ella la autoría de textos que manejan con los niños. Estudios como los de Ambrós (2014) o Lorenzo y Rodríguez (2015) refuerzan esa impresión relativa a la atracción y tendencia de los más pequeños por el acceso al conocimiento de una manera distinta.

Situaciones de ese tipo van alejando a la población del sentido cultural del conocimiento y, lógicamente fragmentan la información, originando lagunas en el pensamiento de los individuos. Este hecho, desde nuestro punto de vista, tiene dos aproximaciones. La primera tiene que ver con lo tradicional, vincularía lo acontecido con la crisis lectora de la que hemos hablado previamente. Pero en esa situación trascendería el nivel lector y se convertiría en una crisis de identidad cultural unida a la, en ocasiones, escuchada, crisis de las humanidades.

Previamente a la implementación de internet, ya se apreciaba una relevancia de lo tecnológico frente a lo humanista, propiciando una peligrosa dicotomía. En este caso, la pérdida de referentes culturales completos se consideraría un peligro social $y$, por consiguiente, una urgencia social que subsanar.

En el segundo de los casos, la visión sería distinta, debido a que atendería a un fenómeno de evolución de pensamiento, de pragmatismo o, en definitiva de ajuste a las condiciones sociales que rodean a la persona. Si esta tiene la información a su alcance mediante la utilización de un medio tecnológico, no parece tan necesario que tenga que hacer uso de su habilidad lectora para poder confeccionar un determinado bagaje cultural. Es más, podría suceder que por imposición de la masa social, los textos audiovisuales que suponen adaptaciones, podrían ser más halagados que las historias originales en las cuales se inspiran. También, como hemos expresado, si la alfabetización temprana tiene que ver con la información que se obtiene de los formatos audiovisuales, las personas educadas de ese modo confiarán más en los detalles que les ha proporcionado este medio, que en aquellos que no conocen pese a que la convención social pueda reclamar que, culturalmente, el correcto es el que tiene que ver con la cultura escrita.

Planteados los dos casos, parece oportuno reflexionar en torno a la existencia y pertinencia de los dos modelos en el contexto social que trasciende a los niños. 
Como nos indican Apolo, Bayés y Hermann (2015), las aportaciones audiovisuales permiten pensar en cambios de tipo pedagógico, ya que, las influencias que reciben los estudiantes son variadas y, principalmente, proceden de medios tecnológicos y del contenido que estos comportan. Así mismo, es evidente que el ámbito audiovisual se encuentra explorando un mercado relacionado con la Literatura infantil y esto queda reflejado, sobre todo, en las aplicaciones que, derivadas de libros o inspiradas en textos se muestran para el público de estas edades (García-Rodríguez y GómezDíaz, 2017). El carácter interactivo que estas tienen implica una motivación especial para los niños y contrasta con los textos escritos. Afortunadamente, la demanda de estos últimos no decae y las editoriales siguen publicando historias en forma de álbumes ilustrados o, por ejemplo, cuentos. Este escenario hace que nos planteemos la forma de pensar y de comprender los textos por parte de las futuras generaciones, la forma de concebir las historias (no tanto personal, puesto que el imaginario viene impuesto por el global uniforme). Consideramos que su opción por los medios tecnológicos será evidente aunque sigan manejando los textos escritos presentados en formato físico. En materia de preparación de profesores López-Valero y Encabo (2015) nos planteaban estas nuevas situaciones derivadas de la presencia audiovisual y cómo podrían afectar al pensamiento y competencia literaria de los docentes. Por esa razón tenemos que reflexionar sobre si va a producir una nueva forma de pensamiento derivada de ese uso y, sobre todo, de la adquisición de una competencia enciclopédica probablemente parcelada.

\section{LA ADQUISICIÓN DE CONOCIMIENTO PARCELADO ¿ UNA NUEVA FORMA DE PENSAMIENTO?}

El anterior recorrido argumentativo nos conduce a reflexionar sobre el ámbito educativo y cómo, tal vez, este no esté avanzando al mismo ritmo que las cuestiones sociales. La cantidad de tiempo que las personas están expuestas a los medios digitales es equiparable sino superior a la que emplean con los textos que clásicamente se han presentado en formato físico. Es importante remarcar este hecho, ya que, la interpretación de la realidad está estrechamente vinculada al medio por el que se transmite la información. Cierto es que no podemos afirmar que las preocupaciones del ser humano hayan variado de manera significativa pero el modo en que se envuelven tales ideas y la forma en que se accede a ellas ya no se puede catalogar como algo lineal.

La presentación que desde un texto en formato físico se realiza de la información tiene que ver con una progresión lineal en la que, necesariamente, necesitamos comenzar por un punto de partida para, de manera progresiva, avanzar por las distintas ideas propuestas por el autor del texto, finalizando en otro punto que se caracteriza por sugerir una serie de conclusiones. El motivo de esa disposición puede concernir a las limitaciones del formato papel que no se caracteriza por mostrar gran flexibilidad tanto a autores como a lectores. En la actualidad, la eclosión de los formatos digitales -cuyo límite no parece haberse alcanzado- provoca que la información ya no se presente necesariamente de una manera lineal sino que la circularidad $y$, sobre todo, los saltos textuales, sean posibles. Tanto los hipertextos como los hipervínculos rodean 
la actividad de percepción y de interpretación de las personas, motivando, sin duda, que la forma de pensar se vea afectada. Galán (2012) y también Hermann (2012) ya comentan en su texto qué influencia tiene la presencia de tales hipertextos en el desarrollo del pensamiento y cómo debemos tener en cuenta tales transformaciones.

El impacto es dual, es decir, tiene beneficios pero a la vez plantea incertidumbres y posibles perjuicios. Poder acceder a múltiples ideas e información partiendo de un determinado núcleo expande de manera significativa el modo de ver y apreciar la realidad de la personas y, además, permite que en un tiempo más reducido que antaño se pueda encontrar información importante que la persona busca. Como contraprestación hay que mencionar, lo que se apuntaba en algunas de las líneas previas de este apartado, y es que el modelo escolar no ha tenido variaciones significativas. La forma en que está concebida la educación del siglo XXI no se diferencia demasiado de los planteamientos que se producían décadas atrás y los cambios tecnológicos acaecen ahora más rápido que nunca en la historia de la humanidad.

Las formas que se enseñan en el ámbito escolar están más próximas a los usos que se hacían en el pasado -aunque se sigan haciendo en el presente, precisan ser actualizados- y esto genera cierta desincronización entre métodos y contenidos. No hay una correspondencia directa entre la búsqueda directa de información que pueden hacer los niños y jóvenes con una tableta, un móvil o un ordenador, con lo que suelen llevar a cabo en el aula usando los libros de texto. Probablemente sea una búsqueda que a veces no es razonada pero es la que suelen aplicar. La reflexión nos debe conducir a una pregunta clave: ¿es ese modo de pensar el que se ha enseñado tradicionalmente en las instituciones educativas?, ¿tiene que ver con esa descripción patológica del pensamiento lineal de la que nos habla Buela (2007)? Desde estas líneas preferimos hablar de una forma de pensar distinta, ni peor ni mejor, simplemente diferente.

La falta de sincronía entre lo social y lo educativo ha motivado que no se aprecie armonía entre ambas situaciones, generando malestar por parte de los enfoques tradicionales que suelen acusar a las nuevas generaciones de no poseer un conocimiento deseable. Hay que indicar al respecto que, el mundo digital ha facilitado el acceso a una infinidad de información que, en muchas ocasiones, queda como un océano de ideas y hechos, ya que, es complicado trazar al alumnado caminos de acceso a tal información para que esta se convierta en conocimiento. Parte de la atribución del hastío ante los contenidos del ámbito escolar que muestra el alumnado puede conferirse a la falta de correspondencia entre lo realizado en el contexto formal y lo que se hace en el informal.

La formación inicial y continua del profesorado tampoco encuentra una solución a estos escenarios, ya que, al formar parte del sistema educativo, se encuentra sujeta a los presupuestos del funcionamiento del mismo. Es decir, si la filosofía educativa no se altera, los pequeños esfuerzos de actualización no son significativos; y, como hemos señalado previamente, el entramado de los libros de texto supedita la enseñanza reglada a unas condiciones que no terminan de ser compatibles con las tendencias audiovisuales que niños y jóvenes experimentan en su tiempo de ocio y familiar. Reflexionemos a continuación con respecto de cómo queda afectada la Literatura 
infantil en el marco de esta atmósfera de comportamiento social que hace que convivan materiales digitales y formatos en texto físico.

\section{Estética actual de la percepción i HaY NeCESIDAd de UN CANON LITERARIO?}

La brecha generacional es algo palpable social y educativamente y también afecta al contexto de la Literatura infantil. Tradicionalmente ha habido debate sobre si existe o no un canon determinado dentro de este tipo de literatura. Pese a que hay estudiosos del tema que lo han investigado, no ha habido unanimidad en torno a tal hecho. Es más, desde estas líneas nos planteamos si con el panorama social que se detecta hay una necesidad de un canon literario o, si por el contrario, la heterogeneidad de contenidos no haría falta o sería de tipo ecléctico tendente a lo ingobernable.

¿Necesitan los niños un canon literario relacionado con la Literatura infantil? Lógicamente la respuesta que tiene que ver con esta cuestión está relacionada con el mundo educativo. La presencia de dicho canon tiene que ver con una selección de lecturas que se rigen por unas determinadas convicciones y valores. Al respecto Skoda (2012) ya nos indica que el canon suele estar influido por la crítica literaria, luego por aquellos textos que, a veces, son mejor valorado socialmente. El pensamiento de los niños queda afectado en función de los textos a los que se expone mediante el acto de leer. Así, su competencia literaria suele estar notablemente afectada por el canon (Bermudez, 2012). El modelo tradicional permitía la presencia de un determinado canon y en función del mismo se han efectuado diversas aproximación al mismo, por ejemplo, la de Tejerina (2004). Pero, las características de la sociedad actual donde la mezcla de medios audiovisuales con textos físicos hace que los niños accedan a información y contenidos diversos que no pueden agruparse en un mismo nivel, hace replantearse la presencia de un determinado canon en la educación de los niños. En el caso referido a que quisiésemos contemplar la presencia de dicho canon, ¿quién lo dictaría? Probablemente las marcas, youtube o las propias aplicaciones. Los canales temáticos dedicados a niños que se muestran en las tabletas que se encuentran a la elección de niños para que puedan ir accediendo a información infantil (programas, series, juegos) en muchas ocasiones siguen un patrón comercial encumbrando a personajes que van a generar una serie de merchandising con el que las empresas puedan comerciar. En ese caso observaríamos cómo el condicionante del canon sería económico. Al respecto, son interesantes las contribuciones de Campos y García Rivera (2017) o García Única (2017) cuando mencionan vertientes actuales que tendrían que ser tenidas en cuenta para ser parte de ese posible canon literario que afectaría a la Literatura infantil. Entre ellas se situarían la ecocrítica o el ecologismo, cuyo papel podría parecer lejano a los modelos referentes para el alumnado pero que analizados con profundidad se ubican en un lugar mucho más cercano a los intereses de este de lo que tradicionalmente se ha considerado.

Aunque, exactamente no podríamos hablar de canon, ya que, los contenidos tienden a ser tan heterogéneos que no siguen una lógica más allá de la económica que ya hemos mencionado. Por tanto, ¿tiene sentido hablar de canon en un contexto de globalización? No parece que el canon literario pueda ser armado, aunque el canon 
tradicional sí que va a seguir vigente. Es decir, los textos clásicos, como cuentos o novelas infantiles que han pervivido durante décadas, van a continuar pero, no va a ser sencillo identificar un canon puro. Las razones principalmente van a estar unidas a la irrupción audiovisual. La mezcla de formatos consultados por parte de los niños hará que la literatura no sea algo fácilmente distinguible de lo aportados digitalmente. ¿Cómo consigue distinguir el niño un texto literario de un material de lectura que sea meramente la transposición de una serie de televisión o de una película de animación? El reconocimiento de lo visionado hace que el niño disfrute del manejo de ese texto en formato físico pero realmente no ha sido concebido como obra literaria sino como un producto que permite prolongar el éxito que digitalmente ha tenido.

Esa situación nos hace cuestionarnos si es posible la presencia social de un canon híbrido. Este contemplaría las producciones procedentes de la Literatura infantil que se transmiten a los niños vía texto físico y, por otra parte, añadiría las producciones audiovisuales que van conformando el discurrir de los más pequeños. Es complicado poder configurar un bosquejo de canon híbrido pero sí que podemos mencionar qué elementos podrían formar parte de él. Así, por ejemplo, no debemos olvidar la aportación de Tejerina (2004) cuando selecciona cien obras clave de la Literatura infantil. A ellas habría que sumar las manifestaciones audiovisuales que culturalmente tienen una importancia incidencia. Por ello, la Factoría Disney/Pixar se situaría en uno de los lugares trascendentes de dicho canon. Para complementarlo canales temáticos como por ejemplo youtube kids (https://kids.youtube.com/) o cualquiera de aquellos que en su artículo revisan Fernández Gómez y Díaz Campo (2014), Disney Channel, Boing o Neox. La gran variedad de recursos audiovisuales que los niños tienen a su disposición afecta a los modelos de transmisión cultural y esa sería la razón que daría cabida a estas manifestaciones en un hipotético canon híbrido.

En la aludida conformación parcelada del pensamiento enciclopédico también se detecta entre los niños dificultad para ubicar en una línea temporal las producciones que percibe o para asociar determinados personajes que puedan estar en historias conectadas. Por ello, parece pertinente como nos indican Dueñas, Tabernero, Calvo y Consejo (2014) replantear la educación literaria incluyendo en ella los nuevos retos; entre ellos se encuentra analizar el canon y, por supuesto, realizar la propuesta relativa a aunar en un mismo universo producciones literarias y audiovisuales, ya que los receptores son partícipes de ambas y correspondería al profesorado saber encauzar ambas modalidades para que el pensamiento de los niños sea coherente y adaptado a los tiempos en los cuales habitan. Para ello, el profesorado debe estar actualizado y conocer las tendencias tanto en Literatura infantil publicada por las editoriales como por los canales que tienen que ver con la producción audiovisual destinada a los niños.

\section{Conclusiones}

Las ideas que alcanzamos tras el desarrollo de esta contribución tienen que ver con la descripción del espectro social en el que los docentes, bibliotecarios, educadores y alumnado realizan sus acciones cotidianas. El espacio compartido entre nativos y residentes digitales ha motivado que la lectura quede cuestionada, hacién- 
donos reflexionar sobre si el concepto y sus implicaciones sufren una crisis o no. La relatividad es la que proporciona respuesta a tal pregunta, ya que, dependiendo del prisma desde el que enfoquemos la descripción de la realidad, atenderemos a una percepción u otra. Desde una óptica tradicional, la crisis sería un hecho tangible pero, desde un sentido evolutivo, en el que se atiende a las características sociales y de la población, podríamos hablar de nuevos contextos y nuevas situaciones. Estas, tendrían como aspiración la coexistencia de los formatos físicos de lectura que son tradicionales y los formatos audiovisuales, con el correspondiente impacto que estos tienen en la conformación del pensamiento de la ciudadanía contemporánea.

Indagar en los aspectos de conocimiento cultural que las personas poseen con respecto de la Literatura y, más concretamente, de la Literatura infantil, supone encontrar una competencia enciclopédica que incluye saberes procedentes de los formatos audiovisuales, si bien acontece la paradoja concerniente a que las personas confundirán esa procedencia con la de la cultura letrada. La encrucijada temporal en las que nos encontramos motiva que esas respuestas encuentren justificación en la necesidad de tener un respaldo académico-cultural al proceder la información de tal contexto, pero que en el fondo también hallen contradicción en la información, al haberse modificado esta por el efecto de las adaptaciones que suponen las versiones fílmicas. Por tanto, encontramos una población con competencia enciclopédica más que literaria $y$, en ese conocimiento también hallamos lagunas, motivadas principalmente por la cantidad y dispersión de conocimiento al que están expuestas.

Como reflexión importante tenemos que plantearnos si este acceso al conocimiento de una forma distinta propicia o no una nueva forma de pensamiento. La fragmentación de las ideas, del conocimiento, afecta a lo literario, y en lo que concierne a la Literatura infantil, se refleja en cierta mezcla de personajes, acciones y argumentos. Los motivos de inspiración de las factorías (i.e. Disney/Pixar) si bien incluyen alusiones clásicas apuestan por la creación de nuevos personajes o la transformación de esos protagonistas clásicos. Esta transmisión fílmica moldea el pensamiento de los receptores que, de un modo u otro, estereotipan la Literatura infantil, englobándola en ese espectro audiovisual. El texto impreso queda subyugado a la cultura de la imagen y, sobre todo, de la animación. Por ello, otra de los interrogantes de los que dejamos constancia en este texto es el que se refiere a la necesidad o no de un canon literario en Literatura infantil. Esa mezcla de formatos hace que sea muy complicado poder definir dicho canon.

Si previamente ya era dificultoso concretar tal canon, si tomamos como referencia este enfoque comprensivo que aúna formatos, tendremos que dudar de la posible existencia de él, o bien pensar en un canon híbrido muy flexible, en el que probablemente, la influencia de los mercados, con personajes populares y su merchandising asociado, se conviertan en los referentes de la Literatura infantil. La tarea docente es compleja pero solo ante los retos se avanza. Niños y adolescentes nativos digitales que pierden la capacidad de concentrarse en la tarea de leer un texto extenso y crear una imagen mental y única y personal de lo que leen. Llevémosles al texto impreso desde la pantalla a las fuentes, a las obras sin hibridación. También a los clásicos, y a la misma vez, aprovechemos como docentes 
esa perspectiva suya de utilizar de otra manera el texto impreso. Ampliemos su conocimiento de los distintos sistemas de significación (interactivo, audiovisual, escrito). Veamos ese collage en relación al canon, géneros y demás y utilicémoslo para sorprenderles con las obras originales. El medio social hoy en día acontece a gran velocidad. No podemos educar al margen de lo que en él acaece, pero sí podemos usarlo para mejorar la educación y no perder por el camino ni el interés ni la motivación del alumnado.

Debemos concluir este artículo insistiendo en la necesidad referida a que los profesionales del mundo educativo y, más concretamente del ámbito lingüístico y literario reflexionen en torno a la idea del cambio en la concepción de la Literatura infantil, sobre cómo esta noción está condicionada por la condiciones sociales y culturales, elementos que hacen que la definición deba variar (López Valero, Hernández Delgado y Encabo, 2017). Además de esa variación, hay que insistir en la consideración de estrategias distintas que permitan un acceso más motivador al conocimiento (García González y Pérez Martín, 2016). Estos dos puntos son importantes si abordamos la idea que concierne a la conformación del pensamiento de las personas en el siglo XXI. Este se nutre de diferentes estímulos, no siendo la letra impresa la única vía de conocimiento. Por consiguiente, este texto pretende ser inspirador para que los docentes y educadores tengan en cuenta los contextos educativos y sociales en los que el alumnado está inserto para que intenten ajustar las estrategias de enseñanza a esas circunstancias.

\section{REFERENCIAS BIBLIOGRÁFICAS}

Alonso, C. (2015). Aproximaciones teóricas en torno al debate de la literatura en la escuela. Sophia, 11(2), 115-127.

Ambrós, A. (2014). Hipertextos fragmentados. Textos de didáctica de la lengua y la literatura, 65, 73-82.

Apolo, D. E., Bayés, M. y Hermann, A. (2015). Cambios educativos en los procesos de lectura digital: la pedagogía del ciberespacio como estrategia de procesamiento de contenidos en la era de internet. Redes.com: revista de estudios para el desarrollo social de la comunicación, 12, 222-249. https://doi.org/10.15213/redes. n12.p222.

Basanta, A. (2017). Leer contra la nada. Madrid: Siruela.

Bermúdez, M. (2012). Competencia literaria y literatura infantil y juvenil: una lectura desde el canon. Textos de didáctica de la lengua y la literatura, 60, 92-101.

Buela, A. (2007). El pensamiento lineal: otra patología del pensamiento único. Altar Mayor, 112, 671-675.

Campos, M. y García Rivera, G. (2017). Aproximación a la ecocrítica y a la ecoliteratura: literatura juvenil clásica e imaginarios del agua. Ocnos, 16(2), 95-106. https://doi.org/10.18239/ocnos_2017.16.2.1511.

Cerrillo, P. (2016). El lector literario. México: Fondo de Cultura Económica. 
Dueñas, J. D., Tabernero, R., Calvo, V. y Consejo, E. (2014). La lectura literaria ante nuevos retos: canon y mediación en la trayectoria lectora de futuros profesores. Ocnos: revista de estudios sobre lectura, 11, 21-43. http://doi.org/10.18239/ ocnos_2014.11.02.

Edwards, L. H. (2012). Transmedia storytelling, corporate synergy, and audience expression. Global Media Journal, 20(12), 1-12.

Federación de Gremios de Editores de España (2018). Barómetro de hábitos de lectura y compra de libros en España 2017. Madrid: Federación de Gremios de Editores de España. Recuperado de http://federacioneditores.org/index.php.

Fernández Gómez, E. y Díaz Campo, J. (2014). Los canales temáticos infantiles y juveniles en Facebook: análisis de los perfiles de Disney Channel, Boing y Neox. Comunicación y hombre, 10, 179-194

Galán, J. F. (2012). El hipertexto paralelo y el desarrollo del pensamiento. Sophia: colección de Filosofía de la educación, 12, 41-51.

García González, S. y Pérez Martín, J. M. (2016). Enseñanza de las Ciencias Naturales en Educación Primaria a través de cuentos y preguntas mediadoras. Revista internacional de investigación e innovación en Didáctica de las Humanidades y las Ciencias, 3, 101-122.

García Padrino, J. (2005). La promoción de la lectura: una permanente tarea educativa. Revista de Educación, 1, extra, 37-51. https://doi.org/10.4438/1988-592X0034-8082-RE.

García-Rodríguez, A.y Gómez-Díaz, R. (2017). Literatura digital infantil y juvenil en tabletas y smartphones: una oportunidad para lograr nuevos lectores. Anuario ThinkEPI, 11, 167-174.

García Única, J. (2017). Ecocrítica, ecologismo y educación literaria: una relación problemática. Revista interuniversitaria de formación de profesorado, 31 (3), 79 91.

Hermann, A. (2012). Ficcionalización, pensamiento, lenguaje y nuevas narrativas visuales. Sophia: colección de Filosofía de la educación, 12, 107-121.

Jenkins, H. (2008). Convergence Culture. La cultura de la convergencia de los medios de comunicación. Barcelona: Paidós.

Koenitzm, H., Ferri, G., Haahr, M., Sezen, D., Sezen, T. I. (Eds.) (2015). Interactive digital narrative: history, theory and practice. New York, Londres: Routledge.

López-Valero, A. y Encabo, E. (2015). Cuando la competencia literaria y la competencia enciclopédica se solapan. Un problema en la formación del profesorado. Lenguaje y textos, 41, 27-34. https://doi.org/10.4995/lyt.2016.5820.

López Valero, A., Hernández Delgado, L. y Encabo, E. (2017). El concepto de literatura infantil. Un estudio de caso con maestros en formación inicial. Ocnos, 16(2), 37-49. https://doi.org/10.18239/ocnos_2017.16.2.1400. 
Lorenzo, L. y Rodríguez, B. M. (2015). La intertextualidad en los textos audiovisuales: el caso de Donkey Xote. Ocnos: revista de estudios sobre lectura, 13, 117-128. http://doi.org/10.18239/ocnos_2015.13.07.

Martínez Ezquerro, A. (2016). El método de cooperación interpretativa como estrategia lectora. Álabe, 14. https://doi.org/10.15645/Alabe2016.14.1.

Quiles, M. C. Palmer, I. y Rosal, M. (2015). Hablar, leer y escribir. El descubrimiento de las palabras y la educación lingüística y literaria. Madrid: Visor.

Sánchez Morillas, C. M. (2013). Crisis del personaje. La bruja en la era tecnológica. Tejuelo: Didáctica de la lengua y la literatura, 17, 56-66.

Scolari, C. (2017). El translector. Lectura y narrativas transmedia en la nueva ecología de la comunicación. En J. A. Millán (Ed), La lectura en España. Informe 2017 (pp. 175-186). Madrid: Federación de Gremios de Editores de España/MEC.

Skoda, A. (2012). La literatura infantil y juvenil en los márgenes de la crítica literaria. Gramma, 1(4), 217-222.

Tejerina, M. I. (2004). Los cien libros del siglo XX: el canon literario y la literatura infantil y juvenil. Lazarillo: revista de la Asociación de Amigos del libro infantil y juvenil, 12, 17-25. 